\title{
Banda ancha móvil privada y su interacción con redes de voz de la Policía Nacional
}

\author{
Private Mobile Broadband and its Interaction with Police Voice Networks \\ Banda larga móvel privada e sua interacção com as redes de voz da polícia
}

\author{
Yesid Alexander Ahumada Torres ${ }^{\mathrm{a},{ }^{*}} \mid$ Andrés Saúl Moreno Martínez ${ }^{\mathrm{b}}$ \\ a https://orcid.org/0000-0002-8732-3616 Policía Nacional de Colombia, Bogotá D. C., Colombia \\ b https://orcid.org/0000-0001-8522-1512 Policía Nacional de Colombia, Bogotá D. C., Colombia
}

- Fecha de recepción: 2021-04-02

- Fecha concepto de evaluación: 2021-07-31

- Fecha de aprobación: 2021-09-24

https://doi.org/10.22335/rlct.v14i1.1436
Para citar este artículo / To reference this article / Para citar este artigo: Ahumada-Torres, Y. A., \& Moreno-Martínez, A. S. (2022). Banda ancha móvil privada y su interacción con redes de voz de la Policía Nacional. Revista Logos Ciencia \& Tecnología, 14(1), 87-100.

https://doi.org/10.22335/rlct.v14i1.1436

\begin{abstract}
RESUMEN
Las comunicaciones de misión crítica utilizadas a nivel mundial para la seguridad pública han estado basadas en soluciones de banda angosta, enmarcadas en los diferentes protocolos incluidos en Land Mobile Radio (LMR), como APCO 25, utilizado por la Policía Nacional. Si bien estas tecnologías han sido de gran importancia y cuentan con una madurez de implementación bastante consolidada para la transmisión confiable de voz, tienen limitantes para la transmisión de datos como imágenes y videos, por lo que se hace necesario el aprovechamiento de una tecnología como Long Term Evolution (LTE). Teniendo en cuenta esta necesidad, se llevó a cabo una revisión de la literatura científica-técnica, realizando un análisis del estado del arte de artículos seleccionados de varias bases de datos; asimismo, se determinaron criterios de inclusión y exclusión para el estudio, gracias a lo cual se logró identificar que hoy en día no se tiene la capacidad tecnológica para realizar un desarrollo de una red LTE para seguridad pública; con base en lo anterior, se identificó la necesidad de una implementación tecnológica que garantice la coexistencia de las dos redes LMR y LTE, aprovechando las bondades de cada una de ellas en una suma que incrementa las capacidades tecnológicas en el servicio de policía.
\end{abstract}

Palabras clave: Atribución de frecuencias, comunicación móvil, red de telecomunicaciones, teléfono móvil, transmisión de datos

* Autor de correspondencia. Correo electrónico: yesid.ahumada@campusucc.edu.co 


\begin{abstract}
Mission-critical communications used worldwide for public safety have been based on narrow band solutions, framed in the different protocols included in Land Mobile Radio (LMR), such as APCO 25, used by the National Police. Although these technologies have been of great importance and have a fairly consolidated implementation maturity for reliable voice transmission, they have limitations for the transmission of data such as images and videos, making it necessary to take advantage of technology such as Long Term Evolution (LTE). In view of this need, it was carried out a technical scientific literature review, verifying the state of the art in several databases; likewise inclusion and exclusion criteria were determined for the study, thanks to which it was possible to identify that today there is no technological capacity to develope a LTE network for public safety; based on the above the need for a technological implementation that guarantees the coexistence of the two LMR and LTE neworks was identified, taking advantage of the benefits of each one of them in a sum that increases the technological capabilities in the police service.
\end{abstract}

Keywords: Frequency allocation, mobile communication, telecommunications network, mobile phone, data transmission

\title{
RESUMO
}

As comunicações de missão crítica utilizadas a nível mundial para a segurança pública têm-sido baseadas em soluções de banda estreita, enquadradas nos diferentes protocolos incluídos na Land Mobile Radio (LMR), tais como o APCO 25, utilizado pela Polícia Nacional. Embora estas tecnologias têmsido de grande importância e tenham uma maturidade de implementação bastante consolidada para uma transmissão de voz fiável, têm limitações para a transmissão de dados como imagens e vídeos, pelo que faz-se necessário tirar partido de uma tecnologia como a Long Term Evolution (LTE). Tendo em conta esta necessidade, realizou-se uma revisão da literatura científico-técnica, analisando o estado da arte de artigos seleccionados de várias bases de dados; foram também determinados critérios de inclusão e exclusão para o estudo, graças aos quais foi possível identificar que hoje em dia não existe capacidade tecnológica para realizar um desenvolvimento de uma rede LTE para a segurança pública; Com base no acima exposto, foi identificada a necessidade de uma implementação tecnológica que garanta a coexistência das duas redes LMR e LTE, aproveitando os benefícios de cada uma delas numa soma que aumente as capacidades tecnológicas no serviço de polícia.

Palavras-chave: Atribuição de frequências, comunicação móvel, rede de telecomunicações, telemóvel, telemóvel, transmissão de dados

Para el año 2020, el Ministerio de las Tecnologías de la Información y las Comunicaciones (MinTic) emitió a través de la Agencia Nacional de Espectro (ANE) la Resolución 105, según la recomendación de la Unión Internacional de Telecomunicaciones (ITU). Teniendo en cuenta que este ente regulador es el encargado a nivel mundial de emitir las recomendaciones en cuanto al manejo adecuado del espectro radioeléctrico, se determinó la posibilidad de que los entes territoriales puedan hacer uso de un rango de frecuencias de 380 a $398 \mathrm{MHz}$, con el propósito de realizar la implementación de redes de uso privado para comunicaciones a través de banda ancha móvil para el servicio de seguridad en municipios, con lo cual se busca mejorar la atención de incidentes que afectan a la ciudadanía
(ANE, 2020; Congreso de la República, 2009; Ferrús \& Sallent, 2015b; ITU, 2015).

En la actualidad, la Policía Nacional cuenta con una red LMR en protocolo APCO 25 (Association of Public-Safety Communications Officials-International), la cual Ilega a los 1.103 municipios del territorio colombiano, con más de 45.000 terminales móviles; a través de dichos equipos se atienden los diferentes Ilamados que la ciudadanía hace a los sistemas 123. La introducción de LTE complementará, mas no reemplazará las redes LMR existentes, que seguirán siendo la mejor opción para el servicio de vOz de misión crítica en un futuro cercano, debido a capacidades clave como grupos de trabajo y comunicación punto a punto. Teniendo en cuenta las ventajas que 
presenta una red privada $L T E$, se deben definir objetivos de implementación a corto y largo plazo, con el fin de garantizar el mejor aprovechamiento de estas redes de banda ancha móvil en pro de coadyuvar de manera efectiva a la seguridad en municipios y ciudades por parte de la Policía Nacional. Por esta razón, se requiere no solo de pautas de implementación, sino también de establecer cuál será su interacción con las redes de misión crítica que utiliza la institución, garantizando la interoperabilidad entre las mismas, a partir de la premisa de que en la actualidad las redes de radiocomunicaciones P25 son el soporte tecnológico primordial del servicio de la Policía, a través del cual se lleva a cabo la coordinación de la atención de los casos de policía que se requieran, dando cumplimiento al mandato constitucional enmarcado en el artículo 218 de la Carta magna (Astély et al., 2009; Constitución Política de Colombia, 1991; Departamento Nacional de Planeación (DNP), 2006; Ferrús \& Sallent, 2015a).

En consecuencia, se planteó la siguiente pregunta de investigación: ¿Cuál es la mejor opción de implementación para una red de banda ancha móvil LTE enfocada en la seguridad pública y cuál su modo de interacción con las redes P25 que tiene desplegadas la Policía Nacional? Lo anterior, teniendo como objetivo principal identificar el escenario en el cual se generaría el mayor aprovechamiento de los recursos disponibles, garantizando la coexistencia e interoperabilidad con la red actual de la Policía Nacional, para generar una fusión provechosa de bondades tecnológicas que cada sistema presenta.

Para poder tener un panorama amplio, que permitiera el planteamiento de los parámetros adecuados en la elección de la mejor solución a implementar, se realizó una revisión bibliográfica de artículos de investigación en plataformas de búsqueda como IEEE Xplore y ScienceDirect, que estuvieran relacionados con la verificación y utilidad de redes LTE para la seguridad pública, y en los cuales se evaluara la pertinencia del uso de estos sistemas para la operación de misión crítica, teniendo en cuenta las tecnologías actuales desplegadas para la comunicación de voz, sus características y bondades, al igual que sus limitaciones. Estas últimas pueden ser superadas con la implementación de sistemas que permitan una interoperabilidad adecuada para obtener mayores beneficios, que repercuten en la mejora tecnológica del servicio de policía. Al finalizar se hace una descripción detallada del método mediante el cual se realizó la búsqueda y recolección de los diferentes artículos utilizados en la investigación, así como de los resultados obtenidos, y por último, se muestran las conclusiones a las cuales se pudo llegar gracias a la revisión realizada.

Las categorías de análisis definidas como parte de la investigación para la verificación del contenido del texto, mediante la revisión de la literatura científico-técnica de la declaración PRISMA, fueron: ancho de banda, normatividad y transmisión de información. Estas se eligieron con el propósito de limitar la investigación a los aspectos de interés que se necesitaban verificar, y que son los de mayor relevancia para la solución planteada; otras categorías podrían haber sido incluidas, pero esto habría resultado en una investigación dispersa, que no hubiese permitido llegar a una solución concreta (Barbara, 2004; Moher et al., 2019; Urrutia \& Bonfill, 2010).

\section{Método}

Para tener una síntesis de la información que se encuentra disponible acerca de la utilización de tecnologías LTE para la seguridad pública, y su interoperabilidad con los sistemas que actualmente tienen desplegados los organismos de seguridad, se realizó una revisión sistemática siguiendo los lineamientos de la declaración PRISMA, la cual permite seguir paso a paso la selección adecuada de documentos científicos en una investigación, garantizando un enfoque puntual de los temas que se desean investigar, para así orientar a los investigadores sin que se genere una dispersión en el proceso que impida llegar a una conclusión objetiva sobre el tema que se está investigando. Por lo anterior, para la presente investigación se utilizó dicha declaración con el propósito de garantizar una búsqueda adecuada de documentos que aporten a la consecución y análisis de la información, con base en las tres categorías de análisis (Barbara, 2004; Moher et al., 2019; Urrutia \& Bonfill, 2010).

Se eligió la categoría de ancho de banda dentro de comunicaciones móviles, con base en un enfoque comparativo entre las posibilidades de transmisión de información en banda ancha y banda angosta, que están basadas en la implementación de misión crítica, utilizando tecnologías LTE y LMR, respectivamente. La segunda categoría es la transmisión de información, ya sea voz, video o datos, con cada una de sus limitantes para misión crítica en tecnologías de banda ancha móvil y banda estrecha, así como la posibilidad de mantener la interoperabilidad entre estas dos tecnologías para así garantizar una implementación que permita el despliegue de una tecnología funcional 
en el tiempo. Para la tercera categoría de análisis, es decir la normatividad, se analizaron artículos en el ámbito internacional, para confirmar la normalización y estandarización de los requerimientos técnicos necesarios para lograr el trabajo colaborativo de interoperabilidad entre las dos tecnologías; además de lo anterior, se verificó la normatividad nacional sobre la implementación y el aprovechamiento de LTE y sus beneficios para la seguridad pública.

Con el propósito de obtener información más precisa para la investigación, se definieron criterios de inclusión y exclusión. Dentro de los criterios de inclusión se encuentran: estudios publicados entre los años 20102020, relacionados con sistemas de comunicaciones de misión crítica en banda angosta, sistemas de comunicaciones LTE enfocados a seguridad pública, protocolo de misión crítica APCO 25, interoperabilidad de tecnologías LTE y LMR-P25 para seguridad pública y soluciones de implementación Push to Talk Over Cellular (PoC). Como criterios de exclusión se tuvieron en cuenta temas como: tecnología de banda angosta análoga, estándar Digital Mobile Radio, machine learning, internet de las cosas, comunicaciones satelitales, sistemas de comunicaciones tierra-aire, sistemas de comunicaciones con microondas. Para la búsqueda se determinaron estrategias como: el uso de palabras clave dentro de las que se destacan, mission critical LTE, interoperability LTE y LMR, public safety LTE, mission critical PoC y public safety MCPTT (Kuwadekar \& Al-Begain, 2014).

La revisión de la literatura científico-técnica se realizó mediante la verificación de documentos que aportaban información acerca de implementaciones LTE para seguridad pública, y su interoperabilidad con las redes LMR de banda angosta. En este sentido, se limitó la búsqueda a artículos que no fueran anteriores al 2010, y se encontró que para ese año en países como Estados Unidos ya se investigaba acerca de la estructuración de redes LTE para seguridad pública, en los cuales se mostraban las circunstancias que tendría que sortear una red de estas características. Estos estudios fueron impulsados por lo ocurrido con los atentados del 9/11, donde existieron inconvenientes de comunicación entre las diferentes agencias del estado, debido a que no se contaba con una red unificada que permitiera una adecuada interoperabilidad para atender dicha situación (FirstNet, 2020).

Es necesario resaltar que, para lograr un análisis que permitiera una mayor comprensión de los estándares y los recientes desarrollos de redes de banda ancha móvil junto con su aplicación, se dio prioridad a catorce artículos publicados en los últimos cinco años, lo que permitió evaluar las tecnologías ya empleadas en países con un mayor nivel de desarrollo, y que pueden ser útiles para una implementación adecuada en Colombia.

\section{Figura 1}

Número de artículos publicados por año

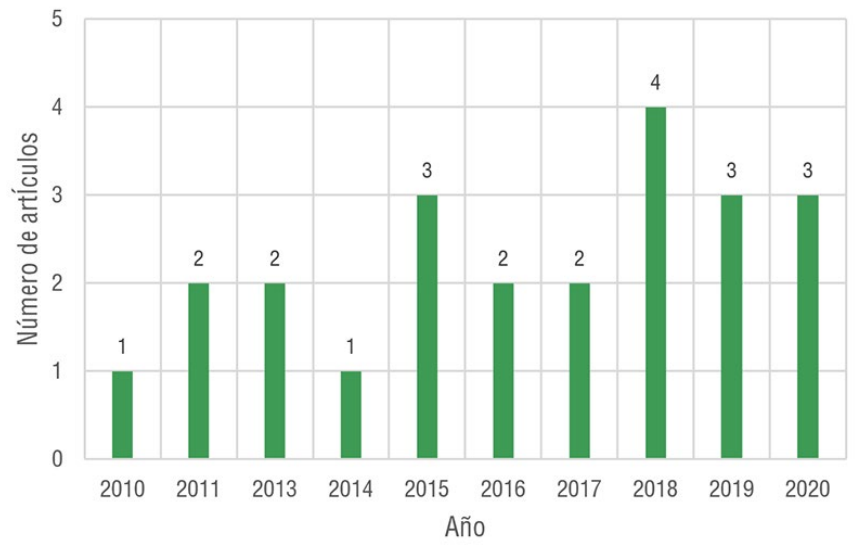

Siguiendo la estructura de la declaración PRISMA para la revisión sistemática de la información, se realizó la selección de documentos con los cuales se planteó la presente investigación.

Figura 2

Diagrama de flujo PRISMA con las diferentes fases de la revisión bibliográfica

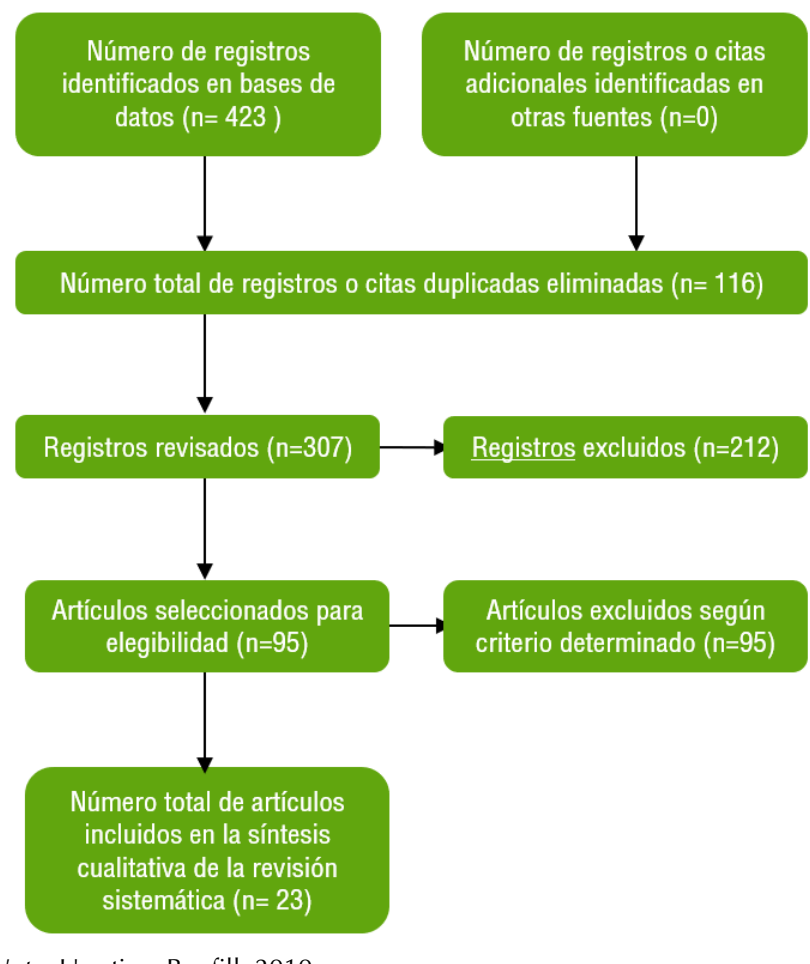

Nota. Urrutia y Bonfill, 2010. 
Dentro del proceso de recopilación de la información se establecieron las siguientes fases, para lograr el objetivo de la investigación:

- Fase 1: se realizó la búsqueda de documentos en bases de datos como IEEE Xplore y ScienceDirect, utilizando palabras claves en inglés como mission critical LTE, interoperability LTE and LMR, public safety LTE, mission critical PoC y public safety MCPTT.

- Fase 2: con base en los resultados, se excluyeron los artículos duplicados; posteriormente, se realizó una selección más detallada a partir de la lectura de los títulos y los resúmenes, con el propósito de identificar si se encontraban dentro de los criterios establecidos.

- Fase 3: a partir del análisis de los artículos seleccionados en la fase dos, se aplicaron las categorías de investigación definidas: ancho de banda, normatividad y transmisión de la información.

- Fase 4: finalmente, se seleccionaron 23 artículos que cumplieron con todos los criterios de la investigación, con lo cual se pretende verificar la información existente y veraz acerca de las implementaciones LTE para seguridad pública y su interacción o interoperabilidad con las redes $\mathrm{P} 25$.

\section{Análisis de datos}

A partir de la información obtenida de cada uno de los artículos como: tipo de revista, categoría de análisis, resumen de la información encontrada, se realizó una base de datos en el software informático Microsoft Excel 2019.

\section{Declaración aspectos éticos}

El presente estudio se realizó respetando los derechos de autor de investigaciones anteriores referente a temas similares, dando los créditos necesarios a cada uno de ellos, citando las fuentes consultadas; del mismo modo, se realizó un trabajo honesto durante la consolidación de la información, con el propósito de obtener un resultado con información verídica, que sea útil para la toma de decisiones sobre la implementación de redes LTE, y su interacción con sistemas P25, que permitan prestar un mejor servicio de seguridad ciudadana.

\section{Resultados}

Dentro del análisis realizado a los 23 documentos que hacen parte de esta investigación, se resalta la calidad de la información recolectada; en este caso, como se obser- va en la figura 3, de los documentos consultados 12 se encuentran en cuartil 1, y solo uno en cuartil 2. Por lo tanto, son documentos con información sólida, sin dejar de lado los análisis hechos a los documentos no indexados, escritos por personal experto en el sector de las tecnologías de la información, sobre la implementación de sistemas de comunicaciones para los entes encargados de la seguridad pública, que cuentan con bases de análisis sólidas.

Figura 3

Categoría de las revistas consultadas

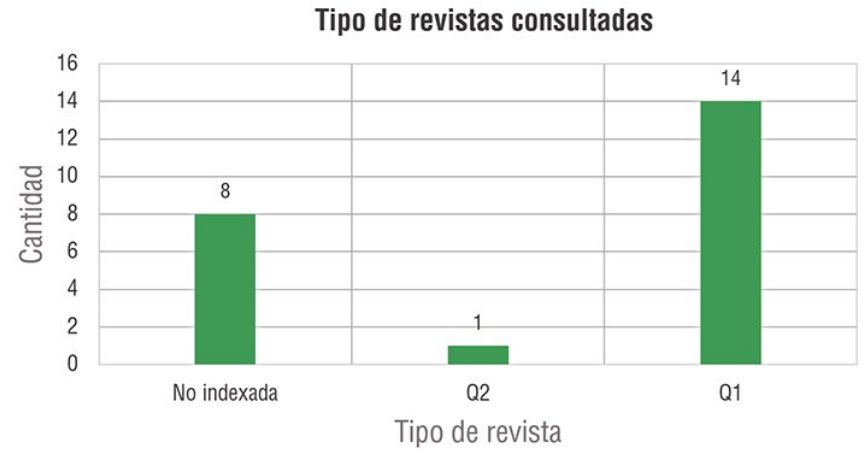

El origen de los documentos analizados lo lidera Estados Unidos, pues este país es uno de los más adelantados a nivel mundial en el desarrollo de tecnología LTE para seguridad pública, y cuenta con la entidad FirstNet, encargada por parte del Gobierno para la aplicación de estas tecnologías. Esta institución fue creada por el Congreso de la República de los Estados Unidos en el año 2012, con el propósito de implementar la ejecución de la red de banda ancha móvil que garantice la conexión de las diferentes unidades estatales encargadas de la seguridad pública y la atención de incidentes.

Son de gran importancia los documentos originados en los Estados Unidos, especialmente porque el sistema LMR P25 que utiliza la Policía Nacional en Colombia sigue un estándar de origen americano, lo cual permite la verificación de documentación donde se evidencian los avances de interoperabilidad entre los sistemas LTE y P25 que pueden ser útiles en una implementación local. En ese sentido, Colombia ya ha dado los primeros pasos en la implementación de redes LTE para seguridad pública, como se demuestra en la selección del análisis de esta investigación: donde en un primer documento analizado hace referencia a una prueba piloto de un sistema LTE realizado en la ciudad de Villavicencio, y el segundo, liderado desde MinTic, señala pautas a los entes territoriales para la implementación de redes privadas de banda ancha móvil, enfocadas, principalmente, en la seguridad de los municipios, con las cuales se 
aprovecha la reciente asignación de espectro para tecnología LTE en la banda de $400 \mathrm{MHz}$.

En la tabla 1 se describen varios aspectos de los trabajos analizados para esta investigación, como por ejemplo, el tipo de publicación, destacándose artículos científicos, conferencias y tesis. Para cada uno de los 23 documentos se realizó una verificación y su correlación con las categorías preestablecidas de ancho de banda, normatividad y transmisión de información.

Tabla 1

Características de tipo y categoría de las investigaciones revisadas

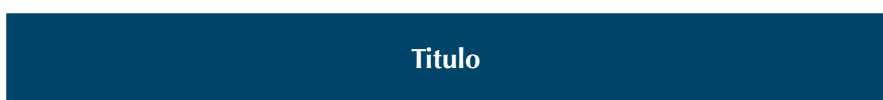

LMR/LTE Interworking Standarsds

Why MCPTTInteroperability Is ImportantW

The Critical Need for Data Interoperability

Análisis de viabilidad técnica y funcional de una red de protección pública y gestión de desastres bajo la tecnología (eLTE) para la Policía Nacional en la ciudad de Villavicencio

Uso del espectro radioeléctrico para servicios de seguridad

Quantifying the costs of a nationwide public safety wireless network

Increasing public safety broadband network resiliency through traffic control

LTE network strategy for Smart City Public Safety

Public Safety Communications Evolution

Surveying pervasive public safety communication technologies in the context of terrorist attacks

Enabling group communication for public safety in LTE-Advanced networks

Consolidation of public safety wireless networks: An options-based economic analysis of numerous scenarios

The importance of LTE interoperability

Gaps in public - Safety Cameras

Efficient and fair Wi-Fi and LTE-U coexistence via communications over content centric networking

LTE techno-economic assessment: The case of rural áreas in Spain

The business case of a network that serves both public safety and commercial subscribers

A Comparative Study of Land Mobile Radio and LTE-based Public Safety Communications

LTE for Public Safety Networks

Evaluation of Datacasting and LTE Integration for Public Safety and First Responder Applications
Medio que contiene el documento

Revista

Revista

Revista

Tesis

Reporte

Revista

Revista

Revista

eBook

Revista

Revista

Revista

Revista

Revista

Revista

Revista

Revista

Revista

eBook

Revista

Revista

Revista

Libro

\section{Categoría}

Ancho de banda, normatividad y transmisión de información

Ancho de banda, normatividad y transmisión de información

Ancho de banda y transmisión de información

Ancho de banda, normatividad y transmisión de información

Ancho de banda, normatividad y transmisión de información

Ancho de banda y transmisión de información

Ancho de banda y transmisión de información

Ancho de banda y transmisión de información

Ancho de banda y transmisión de información

Ancho de banda, normatividad y transmisión de información

Ancho de banda y transmisión de información

Ancho de banda y transmisión de información

Ancho de banda, normatividad y transmisión de información

Ancho de banda, normatividad y transmisión de información

Ancho de banda, normatividad y transmisión de información

Ancho de banda y transmisión de información

Ancho de banda y transmisión de Información

Ancho de banda y transmisión de información

Ancho de banda, normatividad y transmisión de información

Ancho de banda y transmisión de información

Ancho de banda, normatividad y transmisión de información

Ancho de banda y transmisión de información

Mobile Broadband Communications for Public Safety: The Road Ahead Through LTE Technology
Ancho de banda, normatividad y transmisión de información 
En la tabla 2 se describen los demás aspectos definidos para la investigación, tales como: la calificación de los diferentes documentos consultados, su año de publicación, país e idioma, sumando a ello tres variables determinadas, las cuales fueron encontradas de manera transversal en los diferentes documentos: $V_{-} 1$ Interoperabilidad de los sistemas, V_2 Costos y V_3 Seguridad pública.

Tabla 2

Características de cuartil de artículo, año de publicación, país, idioma y variables

\begin{tabular}{|c|c|c|c|c|c|c|c|}
\hline Artículo & $\begin{array}{l}\text { Categoría } \\
\text { documento }\end{array}$ & Año & $\begin{array}{c}\text { País de } \\
\text { publicación }\end{array}$ & Idioma & $V_{-} 1$ & V_2 & V_3 \\
\hline LMR/LTE Interworking Standarsds & No indexada & 2018 & USA & Inglés & SI & SI & SI \\
\hline Why MCPTT Interoperability Is Important & No indexada & 2019 & USA & Inglés & SI & SI & $\mathrm{SI}$ \\
\hline The Critical Need for Data Interoperability & No indexada & 2019 & USA & Inglés & $\mathrm{SI}$ & $\mathrm{NO}$ & SI \\
\hline $\begin{array}{l}\text { Análisis de viabilidad técnica y funcional de una red de protec- } \\
\text { ción pública y gestión de desastres bajo la tecnología (eLTE) para } \\
\text { la Policía Nacional en la ciudad de Villavicencio }\end{array}$ & No indexada & 2017 & Colombia & Español & SI & $\mathrm{SI}$ & SI \\
\hline Uso del Espectro Radioeléctrico para servicios de seguridad & No indexada & 2020 & Colombia & Español & $\mathrm{NO}$ & $\mathrm{SI}$ & SI \\
\hline $\begin{array}{l}\text { Quantifying the costs of a nationwide public safety wireless } \\
\text { network }\end{array}$ & Q1 & 2010 & Reino Unido & Inglés & $\mathrm{SI}$ & $\mathrm{SI}$ & SI \\
\hline $\begin{array}{l}\text { Increasing public safety broadband network resiliency through } \\
\text { traffic control }\end{array}$ & Q1 & 2018 & China & Inglés & $\mathrm{NO}$ & $\mathrm{SI}$ & SI \\
\hline LTE network strategy for Smart City Public Safety & Q1 & 2016 & USA & Inglés & $\mathrm{NO}$ & $\mathrm{SI}$ & $\mathrm{SI}$ \\
\hline Public Safety Communications Evolution & Q1 & 2019 & USA & Inglés & $\mathrm{SI}$ & $\mathrm{NO}$ & SI \\
\hline $\begin{array}{l}\text { Surveying pervasive public safety communication technologies in } \\
\text { the context of terrorist attacks }\end{array}$ & Q2 & 2020 & Países Bajos & Inglés & SI & NO & SI \\
\hline $\begin{array}{l}\text { Enabling group communication for public safety in LTE-advanced } \\
\text { networks }\end{array}$ & Q1 & 2016 & USA & Inglés & $\mathrm{NO}$ & $\mathrm{NO}$ & SI \\
\hline $\begin{array}{l}\text { Consolidation of public safety wireless networks: An options-based } \\
\text { economic analysis of numerous scenarios }\end{array}$ & Q1 & 2011 & USA & Inglés & $\mathrm{NO}$ & $\mathrm{SI}$ & SI \\
\hline The importance of LTE interoperability & No indexada & 2018 & USA & Inglés & $\mathrm{SI}$ & $\mathrm{NO}$ & SI \\
\hline Gaps in public - Safety Cameras & No indexada & 2017 & USA & Inglés & SI & $\mathrm{NO}$ & SI \\
\hline $\begin{array}{l}\text { Efficient and fair Wi-Fi and LTE-U coexistence via communications } \\
\text { over content centric networking }\end{array}$ & Q1 & 2020 & Países Bajos & Inglés & $\mathrm{SI}$ & $\mathrm{NO}$ & $\mathrm{NO}$ \\
\hline LTE techno-economic assessment: The case of rural áreas in Spain & Q1 & 2015 & Reino Unido & Inglés & $\mathrm{SI}$ & $\mathrm{SI}$ & NO \\
\hline $\begin{array}{l}\text { The business case of a network that serves both public safety and } \\
\text { commercial subscribers }\end{array}$ & Q1 & 2011 & Reino Unido & Inglés & SI & $\mathrm{SI}$ & SI \\
\hline $\begin{array}{l}\text { A Comparative Study of Land Mobile Radio and LTE-based Public } \\
\text { Safety Communications }\end{array}$ & Q1 & 2015 & USA & Inglés & $\mathrm{SI}$ & $\mathrm{NO}$ & SI \\
\hline LTE for Public Safety Networks & Q1 & 2013 & USA & Inglés & $\mathrm{SI}$ & SI & SI \\
\hline $\begin{array}{l}\text { Evaluation of Datacasting and LTE Integration for Public Safety and } \\
\text { First Responder Applications }\end{array}$ & Q1 & 2018 & USA & Inglés & $\mathrm{SI}$ & NO & SI \\
\hline Extending the LTE/LTE-A Business Case & Q1 & 2014 & Europa & Inglés & $\mathrm{SI}$ & $\mathrm{NO}$ & SI \\
\hline LTE: The Technology Driver for Future Public Safety Communications & Q1 & 2013 & Europa & Inglés & $\mathrm{SI}$ & $\mathrm{NO}$ & SI \\
\hline $\begin{array}{l}\text { Mobile Broadband Communications for Public Safety: The Road } \\
\text { Ahead Through LTE Technology }\end{array}$ & No indexada & 2015 & España & Inglés & $\mathrm{SI}$ & SI & SI \\
\hline
\end{tabular}

En la tabla 3 se muestran los enfoques que fueron utilizados en los documentos analizados, y se evidencia que se cuenta con tres tipos de enfoques particulares, lo que facilitó la revisión de la literatura científico-técnica. 
Tabla 3

Características de enfoque y autores de cada artículo de investigación

\begin{tabular}{|c|c|c|}
\hline Artículo & Enfoque & Autores \\
\hline LMR/LTE Interworking Standarsds & Cualitativo & Martínez y Monnes (2018) \\
\hline Why MCPTT Interoperability Is Important & Cualitativo & Olbrich (2019) \\
\hline The Critical Need for Data Interoperability & Cualitativo & Voss (2019) \\
\hline $\begin{array}{l}\text { Análisis de viabilidad técnica y funcional de una red de protección pública y gestión } \\
\text { de desastres bajo la tecnología (eLTE) para la Policía Nacional en la ciudad de Villa- } \\
\text { vicencio }\end{array}$ & Mixto & Solís (2017) \\
\hline Uso del Espectro Radioeléctrico para servicios de seguridad & Cualitativo & MinTic (2020) \\
\hline Quantifying the costs of a nationwide public safety wireless network & Mixto & Hallahan y Peha $(2010 ; 2011)$ \\
\hline Increasing public safety broadband network resiliency through traffic control & Mixto & Rouil et al. (2018) \\
\hline LTE network strategy for Smart City Public Safety & Cualitativo & Raza (2016) \\
\hline Public Safety Communications Evolution & Cualitativo & Abdulrahman (2020) \\
\hline $\begin{array}{l}\text { Surveying pervasive public safety communication technologies in the context of } \\
\text { terrorist attacks }\end{array}$ & Cualitativo & Masood et al. (2020) \\
\hline Enabling group communication for public safety in LTE-advanced networks & Cuantitativo & Prasad et al. (2016) \\
\hline $\begin{array}{l}\text { Consolidation of public safety wireless networks: An options-based economic analysis } \\
\text { of numerous scenarios }\end{array}$ & Cuantitativo & Lee $(2011)$ \\
\hline The importance of LTE Interoperability & Cualitativo & Magnnusen (2018) \\
\hline Gaps in public - Safety Cameras & Cuantitativo & Pinson (2017) \\
\hline $\begin{array}{l}\text { Efficient and fair Wi-Fi and LTE- } U \text { coexistence via communications over content cen- } \\
\text { tric networking }\end{array}$ & Cuantitativo & Chi et al. (2020) \\
\hline LTE techno-economic assessment: The case of rural áreas in Spain & Cuantitativo & Ovando et al. (2015) \\
\hline $\begin{array}{l}\text { The business case of a network that serves both public safety and commercial subs- } \\
\text { cribers }\end{array}$ & Cuantitativo & Hallahan y Peha $(2010 ; 2011)$ \\
\hline A Comparative Study of Land Mobile Radio and LTE-based Public Safety Communications & Cuantitativo & Kumbhar y Guvenc (2015) \\
\hline LTE for Public Safety Networks & Cualitativo & Doumi et al. (2013) \\
\hline $\begin{array}{l}\text { Evaluation of Datacasting and LTE Integration for Public Safety and First Responder } \\
\text { Applications }\end{array}$ & Cualitativo & Luu et al. (2018) \\
\hline Extending the LTE/LTE-A Business Case & Cualitativo & Ferrús y Sallent (2014; 2015a; 2015b) \\
\hline LTE: The Technology Driver for Future Public Safety Communications & Cualitativo & Ferrús et al. (2013) \\
\hline $\begin{array}{l}\text { Mobile Broadband Communications for Public Safety: The Road Ahead Through LTE } \\
\text { Technology }\end{array}$ & Mixto & Ferrús y Sallent (2014; 2015a; 2015b) \\
\hline
\end{tabular}

\section{Discusión}

Con el aval que dio en el 2009 el National Public Safety Telecommunications Council (NPSTC) en la utilización de LTE como estándar de banda ancha para las comunicaciones de misión crítica, a fin de que dicha tecnología tenga una capacidad de despliegue que permita la implementación de estándares como Mission Critical Push to Talk (MCPTT), y de fijar cómo debe ser su interacción con los diferentes protocolos que tienen los sistemas de banda angosta de LMR (dentro de los cuales se encuentra P25), entidades como First Responder Network (FirstNet, parte de Nationwide Public Safety Broadband Network), y la National Institute of Standards and Technology, han realizado adelantos para plantear estándares y acelerar el desarrollo de interoperabilidad entre banda ancha LTE y banda angosta LMR. Aunado a lo anterior, la Alliance for Telecommunications Industry Solutions y la Wireless Technologies and Systems Committe iniciaron un proyecto conjunto con la Telecommunications Industry Association 
para soportar FirstNet y lograr así una compatibilidad entre LTE y LMR, en búsqueda de lograr comunicaciones que permitan el intercambio de información a través de voz, datos y video. Una de las necesidades primordiales para soportar una red LTE, con las garantías de funcionalidad necesarias, es la comunicación de dispositivo a dispositivo D2D, utilizando LTE Sidelink, tecnología que se encuentra en desarrollo según recientes datos de 3GPP (2020). La capacidad de resiliencia permite a una estación base, que se encuentre aislada, seguir enrutando llamadas, incluso teniendo en cuenta la baja capacidad de potencia de transmisión de los equipos. Se plantean entonces equipos terminales con mayor potencia en bandas determinadas que garanticen un incremento en el área de cobertura (3GPP, 2013; Camps-Mur et al., 2013; Choi et al., 2019; FCC, 2016; Feng et al., 2013; Ferrús et al., 2013; Ferrús \& Sallent, 2014; FirstNet, 2020; Homeland Security, 2012; Jackson, 2020; Martínez \& Monnes, 2018; Masood et al., 2020; Olbrich, 2019; Yu et al., 2018; Zhang et al., 2016).

Las soluciones de LTE de misión crítica deben contemplar la posibilidad de la ocurrencia de desastres naturales, los cuales pueden generar afectaciones que serán en mayor o menor medida notables dependiendo de la topografía del sector a cubrir, como lo denotan Rouil et al. (2018), concluyendo que un sector plano se podrán restablecer con mayor facilidad las comunicaciones, y donde se da prioridad a los dispositivos con que cuenta el personal de primer respondiente. Otro punto importante a impactar son las áreas rurales, ya que la prelación de cobertura esta en las áreas urbanas, teniendo en cuenta que para los operadores móviles existe un mejor retorno a la inversión, dejando de lado zonas rurales que requieren cobertura de red, como afirman Ovando et al. (2015) convertidas en proyectos inviables económicamente. Es por esto que a la par de los despliegues de tecnología LTE se deben tener en cuenta sistemas de comunicaciones complementarios, que permitirían un acceso más fácil a zonas remotas, como lo relaciona Luu et al. (2018), quien a través de tecnología datacasting pudo realizar transmisión de datos a través de las señales de televisión digital, permitiendo capturar video vía streaming desde dispositivos LTE y haciendo llegar la información a organismos o ciudadanía en general (Engels et al., 2013; Griffith et al., 2015; Marojevic et al., 2018).

Con la sobrecarga que pueden tener las redes LTE, existen alternativas de transferencia de datos inalámbricos, que pueden generar un aprovechamiento del espectro de uso libre para alivianar la carga sobre las frecuencias utilizadas para redes móviles LTE (Chi et al., 2020); sin embargo, han surgido inconvenientes al generar interferencias y traslapos con sistemas $\mathrm{WiFi}$, ya que estos últimos trabajan mayormente en bandas libres.

Las redes LTE permitirán un gran despliegue de sistemas de video, los cuales tendrán la posibilidad de realizar transmisiones en vivo desde el sector donde se presentan los hechos, lo que constituye un factor importante al momento de tomar decisiones (Pinson, 2017), pero existen factores que generan barreras técnicas al momento de transmitir información a los puestos de mando, relacionados muchas veces con aspectos de clima, humo, iluminación, Iluvia, entre otras. La implementación radica en el diseño de algoritmos de video que permitan optimizar la imagen transmitida con la ayuda de sensores de ultrasonido y ópticos, de tal forma que estos puedan sobreponerse entre sí.

De otra parte y con el propósito de asegurar una implementación adecuada que permita una interoperabilidad entre las tecnologías anteriores y nuevas, se debe tener conocimiento de su funcionamiento, como lo plantea Abdulrahman (2020), quien resalta que los sistemas LMR hoy en día continúan siendo la principal ruta de comunicaciones de voz para los diferentes servicios de seguridad pública. Sumado a lo anterior, Doumi et al. (2013) presentan un estudio donde señalan que los sistemas actuales de misión crítica (como P25) tienen un grado importante de madurez en su desarrollo e implementación, permitiendo una robustez que un sistema LTE para misión crítica no tiene, aunque haciendo la salvedad de que un sistema de banda ancha móvil para seguridad pública brindaría muchas más herramientas para una mejor atención de incidentes. Una de las opciones planteadas por varios de los autores e investigados es la implementación de un sistema Push to Talk Over Cellular (PoC), que permite una trasmisión de voz sobre LTE (VoLTE), y que a su vez facilita una implementación de transmisión de voz tal como Radio Over IP (RoIP) (ETSI, 2021; Ferrús et al., 2013; Kumbhar \& Guvenc, 2015; Martínez, 2016; Saafi et al., 2020; Voss, 2019).

Esta migración se debe realizar de una manera organizada, permitiendo una interoperabilidad entre las dos tecnologías que pueda tener la capacidad de compartir información desde cualquier punto en cualquier red (Magnnusen, 2018), para lo cual se plantea el uso de gateways como una forma de estandarización (ETSI, 2021; Ferrús et al., 2013; Kumbhar \& Guvenc, 2015; Martínez, 2016; Saafi et al., 2020; Voss, 2019). 
En el proceso de puesta en marcha de una solución LTE para la seguridad pública se deben tener claros cuáles serán los costos, tanto de implementación como de sostenimiento; del mismo modo, si se da cobertura a una zona pequeña y específica que así lo requiera (o a un área más grande) se deberán tener en cuenta la cantidad de estaciones base requeridas. En un comienzo se debe pensar en una solución a corto plazo, con una implementación virtual de una red LTE para seguridad pública, con el propósito de brindar una solución inmediata (Raza, 2016) y luego estipular una proyección a largo plazo donde se plantean varios escenarios, analizando los costos y beneficios de los diferentes modelos de negocios, así: 1) un sistema de propiedad del usuario, operado por el mismo; 2) uno que sea propiedad del usuario y esté operado por un servidor comercial, y 3) un sistema de propiedad del operador, operado por él mismo, siendo este último modelo el más viable económicamente. Del mismo modo, y con base en el estudio realizado por Hallahan y Peha (2010) se analizaron los pros y contras de una red propia de los entes gubernamentales versus una alianza con un privado que ya tiene un sistema desplegado: se concluye que el hecho de aprovechar los sistemas e infraestructura ya instalados por parte de un operador móvil es de gran utilidad para el desarrollo de una red que permita el acceso a datos móviles por parte del personal de seguridad pública (Ferrús et al., 2013; Ferrús \& Sallent, 2014; Lee, 2011; Prasad et al., 2016).

En el marco de una implementación LTE, se debe plantear una mejor reorganización del espectro, teniendo en cuenta que la tecnología LMR es de banda angosta y utiliza anchos de banda de un máximo de $25 \mathrm{KHz}$; por el contrario, la tecnología LTE opera con una canalización desde los $1.4 \mathrm{MHz}$ y ocupa una mayor cantidad de espacio en el espectro. Para esto se deben evaluar las diferentes determinaciones tomadas en las diferentes regiones del mundo: mientras en Estados Unidos se reservaron frecuencias en la banda de $700 \mathrm{MHz}$, en Europa se ha contemplado no solo esa banda, sino también la banda de los $400 \mathrm{MHz}$; por otro lado, Asia ha implementado la banda de $800 \mathrm{MHz}$, haciendo énfasis en la importancia que tiene una posible reorganización del espectro (Ferrús et al., 2013; Ferrús \& Sallent, 2014, 2015b; Jarwan et al., 2019).

La viabilidad de una asociación público-privada, que sirve a los suscriptores comerciales, y de seguridad pública, desde la perspectiva de un proveedor con fines de lucro, tiene que ver con la implementación de una red inalámbrica de banda ancha que permita fortalecer los sistemas de emergencias y seguridad, para lo cual se debe garantizar la cobertura de red a través de los operadores. Por tanto, se estudia la posibilidad de ofrecer el servicio de transmisión de datos a usuarios gubernamentales para seguridad pública, y a suscriptores que pagarían por hacer uso del mismo, de modo que se incentive a los operadores a consolidar una infraestructura consistente, que permita mejorar los servicios de atención de emergencias (Ferrús \& Sallent, 2014; Hallahan \& Peha, 2011).

La necesidad latente de una implementación LTE de misión crítica en Colombia, para las comunicaciones del personal de seguridad pública, llevó a que se realizara una prueba piloto con la participación de MinTic, en alianza con la ANE, una empresa especialista en sistemas de comunicaciones de banda ancha móvil y la Policía Nacional (Solís Tulande, 2017) en las bandas de frecuencias de $400 \mathrm{MHz}$ y $700 \mathrm{MHz}$, para aplicaciones de Seguridad Pública y Operaciones de Socorro (PPDR, la cual se llevó a cabo utilizando una metodología aplicada, con el propósito de evaluar la funcionalidad y cobertura de las bandas de frecuencias de $400 \mathrm{MHz}$ y $700 \mathrm{MHz}$, así como de verificar la interoperabilidad del sistema LTE con los equipos de misión crítica P25. Esta prueba se realizó durante un periodo de 21 semanas, tiempo en el cual se verificó el funcionamiento, aprovisionamiento de servicios, cobertura y desempeño de la red, concluyendo la viabilidad técnica de una implementación LTE para seguridad pública. Con base en estos desarrollos, el Ministerio de las Tecnologías de la Información y las Comunicaciones promulgó un documento (MinTic, 2020) donde da a conocer a la opinión pública la autorización establecida a través de la ANE (2020) para la utilización de la banda de 380-398 MHz (Comisión de Regulación de Comunicaciones, 2016; MinTic, 2015; The World Radiocommunicacion Conference, 2012).

Es importante pues para una institución como la Policía Nacional de Colombia la implementación de tecnologías que permitan brindar un mejor servicio a la ciudadanía. Teniendo en cuenta esta premisa, a través de la Oficina de Telemática se realizaron los análisis y desarrollos de proyectos que permiten estar a la vanguardia de las soluciones tecnológicas que apoyen el servicio de policía. Por lo anterior, es pertinente una investigación donde se verifiquen las soluciones de banda ancha móvil LTE y su interoperabilidad con sistemas LMR P25 de misión crítica utilizados por la Policía Nacional, para tener los 
fundamentos necesarios que permitan definir cuál será la mejor implementación.

En Colombia ya se ha dado un primer paso, con la emisión de la Resolución 105 de la ANE; ahora se deben generar tanto las políticas como los protocolos de funcionamiento de las redes, a fin de que permitan el aprovechamiento de los beneficios tecnológicos de un sistema LTE con todas las bondades de la banda ancha (transmisión de imágenes, video y datos). De esta manera, se pueden mejorar también las capacidades de los sistemas de comunicación de banda angosta con los que se cuenta hoy en día para las comunicaciones policiales. En la actualidad no existe un desarrollo nativo sobre LTE para misión crítica, como quedó evidenciado en la investigación. Aun así, existe la necesidad urgente de garantizar una aplicación que proporcione cobertura y calidad, haciendo uso adecuado de los recursos económicos disponibles.

\section{Limitaciones}

Dentro del estudio realizado se presentaron varias limitaciones en la selección de los artículos a analizar, tales como el hecho de haber buscado documentación en solo dos idiomas (inglés y español). Si bien se dice que el inglés es la lengua universal, se debe tener en cuenta que hoy en día existen potencias tecnológicas en el ámbito de las comunicaciones de banda ancha móvil cuyo idioma nativo no es la lengua inglesa como es el caso de China y Corea solo para nombrar algunas, países los cuales tienen empresas tecnológicas muy robustas en el ámbito de las comunicaciones móviles (Infobae, 2021).

\section{Conclusiones}

Teniendo en cuenta la revisión de literatura científicotécnica realizada, se puede concluir que en la actualidad no existe un estándar para la tecnología LTE que garantice un sistema de voz de banda ancha en misión crítica, a pesar de los avances logrados por Firstnet en conjunto con 3GPP. Teniendo en cuenta lo anterior, es importante mantener las redes de comunicaciones LMR de banda angosta y de misión crítica en funcionamiento, y buscar al mismo tiempo herramientas que permitan la interoperabilidad entre los sistemas, para así aprovechar las ventajas que tiene cada uno, como transmisión de video e imágenes para LTE y transmisión de voz de misión crítica para LMR. Esta interoperabilidad posibilita una transición entre tecnologías que es totalmente necesaria, pues se prevé que el futuro de las comunicaciones de misión crítica estará enmarcado en la transmisión de banda ancha.
En esta investigación se hizo evidente la necesidad de implementar una red privada LTE para la seguridad pública en Colombia, siguiendo las experiencias planteadas por FirstNet en los Estados Unidos, con el propósito de poder aprovechar todas las bondades de transmisión de datos y video que tiene esta tecnología para la atención de incidentes. Para ello se deben tener en cuenta consideraciones de despliegue, capacidad financiera e interoperabilidad con tecnologías existentes, siendo necesario realizar un análisis exhaustivo a fin de definir cuál será el modelo de negocio a implementar, si se trata de una solución completamente estatal o se buscan alternativas de asociaciones público-privadas, las cuales pueden llegar a ser beneficiosas teniendo en cuenta la infraestructura privada que se encuentra en sitio y su experiencia en el manejo y despliegue de este tipo de redes.

Como resultado se puede concluir que no existe un proyecto nacional para el desarrollo de redes móviles de banda ancha para seguridad pública, pues se ha determinado que cada municipio es el responsable del aprovechamiento de la banda asignada, según la Resolución 105 de la ANE; por lo tanto, no se destina un presupuesto por parte del Estado, ni parámetros básicos de puesta en funcionamiento e interoperabilidad de los posibles sistemas a instalar. En este sentido, es de vital importancia contemplar las necesidades actuales para así realizar una planificación de estructuración, implementación y funcionamiento.

Se requiere entonces la ejecución de un sistema LTE nacional, con capacidad para interconectar las diferentes agencias de seguridad y de atención de emergencias. Este proyecto debe, en la medida de las posibilidades, ser de uso exclusivo de dichas agencias, ya que no parece viable que se compartan recursos con usuarios normales, teniendo en cuenta la disponibilidad y calidad de servicio con que debe contar dicha red. Esta implementación debe tener la capacidad de interconectarse con las redes LMR P25 con que cuenta la Policía Nacional, y para esto se puede hacer uso de una solución PoC enlazada a los sistemas LMR a través de una solución de RolP, la cual realiza la conexión de un gateway entre la nube y un equipo de radio donante y permite la interconexión de equipos PoC que trabajan sobre redes LTE y el sistema de radio P25 de misión crítica de la institución. Se debe aclarar que con esta solución se lograría la interoperabilidad de comunicaciones LTE y LMR P25, pero esta red no sería de misión crítica debido a las limitantes conocidas del sistema LTE. 
Es importante pues aprovechar la tecnología de punta disponible en el mercado, más aún cuando se trata de garantizar la seguridad de la población. Con base en la presente investigación, se requiere un análisis detallado de la normatividad vigente en Colombia sobre la regulación de la telefonía móvil y su comparación con países más desarrollados, en donde se han implementado políticas en las que los organismos de seguridad tienen prelación en el uso de las redes LTE, y con base en lo anterior, implementar los ajustes necesarios en la norma, para aprovechar al máximo los recursos disponibles en pro de la seguridad ciudadana.

\section{Financiación}

La presente revisión sistemática se realizó con recursos propios de los autores. Se hizo uso de equipos de cómputo personales, con su respectivo software de licenciamiento y acceso a internet, para la consulta de las bases de datos que contribuyeron con la construcción del corpus de la investigación.

\section{Agradecimientos}

A la Oficina de Telemática de la Policía Nacional, por permitir el desarrollo de la presente investigación, para poder brindar a través de implementaciones tecnológicas un mejor servicio de policía. A la Universidad Cooperativa de Colombia y a su programa de Maestría en Telemática, por su importante asesoría en la búsqueda y elección de los documentos adecuados para la realización de un artículo bien fundamentado.

\section{Conflicto de intereses}

Los autores manifiestan no tener ningún conflicto de intereses.

\section{Referencias}

3GPP. (2013). Public Safety. 3GPP a Global Initiative. https:// www.3gpp.org/news-events/3gpp-news/1455PublicSafety

3GPP. (2020). 3GPP a global iniative. About 3GPP. https:// www.3gpp.org/about-3gpp

Abdulrahman, Y. (2020). Public Safety Communications Evolution. In IEEE Wiley Telecommunications eBooks (pp. 227-244). Wiley. https://doi. org/10.1002/9781119580157.ch14

ANE. (2020, April 1). Resolucion 105 de 2020. Agencia Nacional Del Espectro. https://normograma.mintic. gov.co/mintic/docs/resolucion_ane_0105_2020.htm
Astély, D., Dahlman, E., Furuskär, A., Jading, Y., Lindström, M., \& Parkvall, S. (2009). LTE: The evolution of mobile broadband. IEEE Communications Magazine, 47(4), 44-51. https://doi.org/10.1109/ MCOM.2009.4907406

Barbara, K. (2004). Procedures for Performing Systematic Reviews. Keele University Technical Report, 33.

Camps-Mur, D., Garcia-Saavedra, A., \& Serrano, P. (2013). Device-to-device communications with WiFi direct: Overview and experimentation. IEEE Wireless Communications, 20(3), 96-104. https://doi.org/10.1109/ MWC.2013.6549288

Chi, K., Du, X., Yin, G., Wu, J., Guizani, M., Han, Q., \& Yang, Y. (2020). Efficient and fair Wi-Fi and LTE$U$ coexistence via communications over content centric networking. Future Generation Computer Systems, 112, 297-306. https://doi.org/10.1016/j.future.2020.05.026

Choi, S. W., Song, Y. S., Shin, W. Y., \& Kim, J. (2019). A feasibility study on mission-critical push-to-talk: Standards and implementation perspectives. IEEE Communications Magazine, 57(2), 81-87. https://doi. org/10.1109/MCOM.2018.1700886

Comisión de Regulación de Comunicaciones. (2016). Resolucion 4972 de 2016. Sistema Único de Información Normativa. http://www.suin-juriscol.gov.co/ viewDocument.asp?ruta=Resolucion/30038120

Congreso de la Republica. (2009). Ley 1341 de 2009. https://www.alcaldiabogota.gov.co/sisjur/normas/Norma1.jsp?i=36913

Constitución Política de Colombia. (2020, September 13). Leyes desde 1992 - Vigencia expresa y control de constitucionalidad [CONSTITUCION_POLITICA_1991]. Secretaria Senado. http://www.secretariasenado.gov.co/ senado/basedoc/constitucion_politica_1991.html\#1

Departamento Nacional de Planeacion. (2006). Implementacion del Sistema Integrado de Emergencias y Seguridad-Sies de Colombia - Documento Conpes 3437. 0-43.

Doumi, T., Dolan, M. F., Tatesh, S., Casati, A., Tsirtsis, G., Anchan, K., \& Flore, D. (2013). LTE for public safety networks. IEEE Communications Magazine, 51(2), 106112. https://doi.org/10.1109/MCOM.2013.6461193

Engels, A., Reyer, M., Xu, X., Mathar, R., Zhang, J., \& Zhuang, H. (2013). Autonomous self-optimization of coverage and capacity in LTE cellular networks. In IEEE Transactions on Vehicular Technology (Vol. 62, Issue 5, pp. 
1989-2004). Institute of Electrical and Electronics Engineers Inc. https://doi.org/10.1109/TVT.2013.2256441

ETSI. (2021). ETSI - THIRD GENERATION PARTNERSHIP PROJECT. THIRD GENERATION PARTNERSHIP PROJECT (3GPP). https://www.etsi.org/committee/3gpp

FCC. (2016, February 3). Narrowbanding Overview. Federal Communications Commission. https://www.fcc. gov/narrowbanding-overview

Feng, D., Lu, L., Yi, Y. W., Li, G. Y., Feng, G., \& Li, S. (2013). Device-to-device communications underlaying cellular networks. IEEE Transactions on Communications, 61(8), 3541-3551. https://doi.org/10.1109/ TCOMM.2013.071013.120787

Ferrús, R., \& Sallent, O. (2014). Extending the LTE/LTE-a business case: Mission- and business-critical mobile broadband communications. IEEE Vehicular Technology Magazine, 9(3), 47-55. https://doi.org/10.1109/ MVT.2014.2333695

Ferrús, R., \& Sallent, O. (2015a). Public Protection and Disaster Relief Communications. Mobile Broadband Communications for Public Safety, 1-48. https://doi. org/10.1002/9781118831243.CH1

Ferrús, R., \& Sallent, O. (2015b). Radio Spectrum for PPDR Communications. Mobile Broadband Communications for Public Safety, 257-315. https://doi. org/10.1002/9781118831243.CH6

Ferrús, R., Sallent, O., Baldini, G., \& Goratti, L. (2013). LTE: The technology driver for future public safety communications. IEEE Communications Magazine, 51(10), 154-161.

https://doi.org/10.1109/MCOM.2013.6619579

FirstNet. (2020). FirstNet Push-to-Talk: LMR Interoperability Option.

FirstNet - Nationwide Network for Public Safety by Public Safety. (2021). FIRSTNET. https://www.firstnet.com/ power-of-firstnet.html

Griffith, D., Rouil, R., Izquierdo, A., \& Golmie, N. (2015). Measuring the resiliency of cellular base station deployments. 2015 IEEE Wireless Communications and Networking Conference, WCNC 2015, 1625-1630. https://doi.org/10.1109/WCNC.2015.7127711

Hallahan, R., \& Peha, J. M. (2010). Quantifying the costs of a nationwide public safety wireless network. Telecommunications Policy, 34(4), 200-220. https://doi. org/10.1016/j.telpol.2010.01.002

Hallahan, R., \& Peha, J. M. (2011). The business case of a network that serves both public safety and commercial subscribers. Telecommunications Policy, 35(3), 250268. https://doi.org/10.1016/j.telpol.2010.12.006

Homeland security. (2012). Nationwide Public Safety Broadband Network. June.

Infobae. (2021). Cuáles son las marcas de celulares más vendidas. https://bit.ly/3vHLZve

ITU. (2015). Resolution 646 (Rev.WRC-15). https://www. itu.int/oth/R0A0600001A/es

Jackson, D. (2020, November 20). Public safety transitioning to LTE-based solutions, but PTT timetable still unclear, speakers say - Urgent Comms. https://bit. ly/3vHLXnc/

Jarwan, A., Sabbah, A., Ibnkahla, M., \& Issa, O. (2019). LTE-Based Public Safety Networks: A Survey. IEEE Communications Surveys and Tutorials, 21(2), 11651187. https://doi.org/10.1109/COMST.2019.2895658

Kumbhar, A., \& Guvenc, I. (2015). A comparative study of Land Mobile Radio and LTE-based public safety communications. Conference Proceedings - IEEE SOUTHEASTCON, 2015-June(June). https://doi.org/10.1109/SECON.2015.7132951

Kuwadekar, A., \& Al-Begain, K. (2014). A real world evaluation of Push to Talk service over IMS and LTE for public safety systems. International Conference on Wireless and Mobile Computing, Networking and Communications, 365-370. https://doi.org/10.1109/ WiMOB.2014.6962196

Lee, S. (2011). Consolidation of public safety wireless networks: An options-based economic analysis of numerous scenarios. Telecommunications Policy, 35(2), 91-101. https://doi.org/10.1016/j.telpol.2010.12.015

Luu, C., Syed, D., Gaither, M., \& Contestabile, J. (2018). Evaluation of Datacasting and LTE Integration for Public Safety and First Responder Applications. 2018 IEEE International Symposium on Technologies for Homeland Security, HST 2018, 1-7. https://doi.org/10.1109/ THS.2018.8574138

Magnnusen, W. (2018). The Importance of LTE Interoperability. MissionCritical Communications, 24-28. MCCmag.com

Marojevic, V., Rao, R. M., Ha, S., \& Reed, J. H. (2018). Performance analysis of a mission-critical portable LTE system in targeted RF interference. IEEE Vehicular Technology Conference, 2017-September, 1-6. https:// doi.org/10.1109/VTCFall.2017.8288187 
Martínez, A. S. (2016). Comunicaciones Críticas de Emergencia Interoperables, Seguras y Globales. Linkedin. https://bit.ly/3bbH10o

Martinez, D., \& P. Monnes. (2018, April). LMR/LTE Interworking Standarsds. Mission Critical Communications, 14-20. https://acortar.link/4IUEP3

Masood, A., Scazzoli, D., Sharma, N., Moullec, Y. Le, Ahmad, R., Reggiani, L., Magarini, M., \& Alam, M. M. (2020). Surveying pervasive public safety communication technologies in the context of terrorist attacks. Physical Communication, 41, 1-23. https:// doi.org/10.1016/j.phycom.2020.101109

MinTic. (2015). Decreto 2434 del 17 de diciembre de 2015. https://normograma.mintic.gov.co/mintic/docs/decre to_2434_2015.htm

MinTic. (2020). Uso del espectro radioeléctrico para servicios de seguridad. https://mintic.gov.co/portal/715/ articles-146317_ciudades_seguras.pdf

Moher, D., Liberati, A., Tetzlaff, J., Altman, D. G., Antes, G., Atkins, D., Barbour, V., Barrowman, N., Berlin, J. A., Clark, J., Clarke, M., Cook, D., D'Amico, R., Deeks, J. J., Devereaux, P. J., Dickersin, K., Egger, M., Ernst, E., Gøtzsche, P. C., ... Tugwell, P. (2019). Preferred Reporting Items for Systematic Reviews and Meta-Analyses: The PRISMA Statement. PLOS MEDICINE, 6(7), 1-6.

Olbrich, E. (2019). Why MCPTT Interoperability Is Important. MissionCritical Communications, 60-65. https://www.rrmediagroup.com/eLearning/frmSignin/ $\mathrm{MCID} / 202$

Ovando, C., Pérez, J., \& Moral, A. (2015). LTE technoeconomic assessment: The case of rural areas in Spain. Telecommunications Policy, 39(3-4), 269-283. https:// doi.org/10.1016/j.telpol.2014.11.004

Pinson, M. (2017). Gaps in public - Safety Cameras. MissionCritical Communications, 30-35. MCCmag.com

Prasad, A., Maeder, A., Samdanis, K., Kunz, A., \& Velev, G. (2016). Enabling group communication for public safety in LTE-Advanced networks. Journal of Network and Computer Applications, 62, 41-52. https://doi. org/10.1016/j.jnca.2015.10.014

Raza, A. (2016). LTE network strategy for Smart City Public Safety. 2016 IEEE International Conference on Emerging
Technologies and Innovative Business Practices for the Transformation of Societies, EmergiTech 2016, 34-37. https://doi.org/10.1109/EmergiTech.2016.7737306

Rouil, R., Garey, W., Gentile, C., Golmie, N., \& Schwinghammer, P. (2018). Increasing public safety broadband network resiliency through traffic control. Digital Communications and Networks, 4(1), 48-57. https://doi.org/10.1016/j.dcan.2017.09.005

Saafi, S., Hosek, J., \& Kolackova, A. (2020). Cellularenabled Wearables in Public Safety Networks: State of the Art and Performance Evaluation. International Congress on Ultra Modern Telecommunications and ControlSystemsandWorkshops, 2020-October,201-207. https://doi.org/10.1109/ICUMT51630.2020.9222459

Solís Tulande, E. (2017). Análisis de viabilidad técnica y funcional de una red de protección pública y gestión de desastres bajo la tecnología (eLTE) para la policía nacional en la ciudad de Villavicencio. Universidad Santo Tomas.

The World Radiocommunicacion Conference. (2012). Studies to support broadband public protection and disaster relief. 1-2. https://www.itu.int/dms_pub/itu-r/ oth/0c/0a/R0C0A00000A0017PDFE.pdf

Urrutia, G., \& Bonfill, X. (2010). Declaracion PRISMA: una propuesta para mejorar la publicación de revisiones sistemáticas y mataanálisis. In Medicina Clínica 135(11), 507-511. http://es.cochrane.org/sites/ es.cochrane.org/files/public/uploads/PRISMA_Spanish.pdf

Voss Britta. (2019). The Critical Need for Data Interoperability. MissionCritical Communications, 52-59. https:// www.rrmediagroup.com/eLearning/eLearning-Registration/MCID/202/userID/9827

Yu, W., Xu, H., Nguyen, J., Blasch, E., Hematian, A., \& Gao, W. (2018). Survey of Public Safety Communications: User-Side and Network-Side Solutions and Future Directions. In IEEE Access 6, 70397- 70425. Institute of Electrical and Electronics Engineers Inc. https://doi.org/10.1109/ACCESS.2018.2879760

Zhang, B., Li, Y., Jin, D., \& Han, Z. (2016). Network Science Approach for Device Discovery in Mobile Deviceto-Device Communications. IEEE Transactions on Vehicular Technology, 65(7), 5665-5679. https://doi. org/10.1109/TVT.2015.2453234 